turnos para estudios complementarios, como cateterismos, estudios electrofisiológicos, imágenes, entre otros.

Si bien el objetivo del trabajo es mostrar un aspecto de los efectos de la pandemia, probablemente se requerirá esfuerzos extraordinarios para recuperar la actividad en relación con la era preCOVID.

Las limitaciones se relacionan con un estudio retrospectivo y con la aplicación de un modelo matemático en el que solo se hace una estimación teórica de los pacientes no operados, y no se evaluaron otros riesgos como oportunidad quirúrgica perdida en grupos de pacientes de mayor riesgo, seguimiento y estudios no realizados.

\section{CONCLUSIONES}

El programa de cirugía cardiovascular tuvo una reducción del $60 \%$ de su capacidad operativa entre los meses de marzo a agosto en comparación con un período similar preCOVID. El tiempo de resolución de la lista de espera generada por la pandemia puede ser, al menos, 10 a 19 meses, aun con la implementación de estrategias de aumento de la oferta y postergando la resolución de pacientes cuya condición permite diferir la cirugía.

\section{REFERENCIAS}

1. HospitalGarrahan. Licencias extraordinarias en el marco de las medidas adoptadas por el gobierno nacional. Intranet, noticias internas. 2020.

2. Althabe M, Rodríguez R, Balestrini M, Charroqui A, et al. Morbilidad en cirugía de cardiopatía congénita en un hospital público en Argentina. Arch Argent Pediatr. 2018; 116(1):e14-8.

3. Magliola R, Laura JP, Capelli H. Situación actual de los niños con cardiopatía congénita en Argentina. Arch Argent Pediatr. 2000; 98(2):130-3.

4. Jenkins KJ. Risk adjustment for congenital heart surgery: the RACHS-1 method. Semin Thorac Cardiovasc Surg Pediatr Card Surg Anпu. 2004; 7(1):180-4.

5. Salenger R, Etchill EW, Ad N, Matthew T, et al The Surge after the Surge: Cardiac Surgery post-COVID-19. Ann Thorac Surg. 2020; 110(6):2020-5.

6. Villa A, Olivetti E, Capelli H. Programa Nacional de Cardiopatías Congénitas: diseño, implementación y resultados a mediano plazo. Rev Argent Salud Publica. 2015; 6(24):39-42.

7. Magliola RH, Althabe M, Moreno G, Lenz A, et al. Cardiopatías congénitas: Resultados quirúrgicos en un hospital público en Argentina. Arch Cardiol Mex. 2011; 81(3):178-82.

8. Stephens EH, Dearani JA, Guleserian KJ, Overman D, et al. COVID-19: Crisis Management in Congenital Heart Surgery. Ann Thorac Surg. 2020; 110(2):701-6.

9. Polo López L, Centella Hernández T, González Calle A, Bautista Hernández V, et al. Cirugía de cardiopatías congénitas en España durante el estado de alarma por COVID-19. Cir Cardiovasc. 2020; 27(4):137-41.

10. Protopapas E, Lo Rito M, Vida V, Sarris G, et al. Early Impact of the COVID-19 Pandemic on Congenital Heart Surgery Programs Across the World: Assessment by a Global Multi-Societal Consortium. World J Pediatr Congenit Heart Surg. 2020; 11(6):689-96.

\title{
Simulación de intubación pediátrica con videolaringoscopio de bajo costo en contexto de pandemia por COVID-19
}

\author{
Simulation of pediatric intubation using a low-cost videolaryngoscope in the \\ setting of the COVID-19 pandemic
}

\author{
Dr. Daniel Rufach ${ }^{a}$ Dra. Silvia Santos ${ }^{b}$ y Dr. Marcelo Terebiznik ${ }^{a}$
}

a. Hospital Interzonal de Agudos "Eva Perón”, San Martín, provincia de Buenos Aires, Argentina.

b. Hospital General de Pediatría "Prof. Dr. Juan P. Garrahan", Ciudad Autónoma de Buenos Aires, Argentina.

Correspondencia:

Dr. Daniel Rufach: jrufach@intramed.net

Financiamiento: Ninguno.

Conflicto de intereses: Ninguno que declarar.

Recibido: 13-9-2020

Aceptado: 1-2-2021

\begin{abstract}
RESUMEN
En pacientes con infección por SARS-CoV-2 la intubación endotraqueal es un procedimiento con riesgo elevado de contagio. La videolaringoscopia complementa la protección del profesional, pero los videolaringoscopios comerciales son caros y no siempre están disponibles en las terapias intensivas pediátricas argentinas. El objetivo fue describir la práctica de intubación en un modelo de cabeza de simulación de lactante con un videolaringoscopio artesanal de bajo costo.

Quince pediatras sin experiencia previa con el dispositivo participaron de una práctica de intubación en una cabeza de simulación con un videolaringoscopio artesanal. El tiempo promedio del primer intento fue de 116,4 segundos (intervalo de confianza del $95 \%$ [IC95\%]: 84,8-148,0) y, el del siguiente
\end{abstract}


fue de 44,2 segundos (IC95\%: 27,7-60,6). El tiempo disminuyó de forma significativa en el segundo intento ( $p: 0,0001$ ). Eldispositivo permitióla intubación exitosa en todos losintentos acortandola duración del procedimiento en la segunda práctica. Palabras clave: videolaringoscopio, intubación endotraqueal, simulación, COVID-19.

http: / / dx.doi.org/10.5546/ aap.2021.270

Texto completo en inglés:

http: / / dx.doi.org/10.5546/ aap.2021.eng.270

Cómo citar: Rufach D, Santos S, Terebiznik M. Simulación de intubación pediátrica con videolaringoscopio de bajo costo en contexto de pandemia por COVID-19. Arch Argent Pediatr 2021;119(4):270-273.

\section{INTRODUCCIÓN}

El nuevo coronavirus (SARS-CoV-2) es un virus de alta contagiosidad que expone a los profesionales de la salud al riesgo de infección. ${ }^{1}$ Uno de los procedimientos que conlleva mayor riesgo de contagio es la intubación endotraqueal. ${ }^{2}$ Los expertos recomiendan el uso de equipo de protección máximo para protegerse de la aerosolización, además de la utilización de secuencia de intubación rápida, ${ }^{3}$ la videolaringoscopia y la práctica con simulación. ${ }^{4-6}$

La videolaringoscopia es una tecnología estándar para el manejo difícil de la vía aérea en niños. ${ }^{7}$ Su uso se ha incrementado en las unidades de cuidados intensivos pediátricos (UCIP) aunque, en comparación con la laringoscopia tradicional, su uso es muy poco frecuente $(89,5 \%$ versus $10,5 \%) .{ }^{8}$ Es una herramienta útil para la enseñanza de la habilidad de intubación, permite guiar el aprendizaje mediante la visualización en un monitor, y es relevante para la calidad de atención y seguridad de los pacientes y para la formación de intensivistas que deben adquirir destreza en el manejo de la vía aérea.

Los videolaringoscopios de uso comercial son caros, con un valor promedio en la Argentina de 370000 pesos, por lo que no están disponibles en todas las UCIP del país. Distintos autores proponen experiencias con videolaringoscopios artesanales de bajo costo. ${ }^{9-11}$

\section{OBJETIVOS}

Describir la práctica de un grupo de pediatras en la intubación de una cabeza de simulación de lactante con un videolaringoscopio artesanal de bajo costo.

\section{MATERIALES Y MÉTODOS}

Estudio descriptivo y observacional.
Durante junio de 2020, médicos pediatras participaron de una práctica de intubación en un simulador de paciente cabeza de lactante marca Laerdal ${ }^{\circledR}$ con un videolaringoscopio artesanal en la UCIP del Hospital Interzonal General de Agudos "Eva Perón" en la provincia de Buenos Aires. Participaron los médicos que concurren al servicio de lunes a viernes, ninguno de ellos con experiencia previa con el modelo de simulación. Realizaron una capacitación previa con una clase teórica y un video tutorial realizado por los autores para familiarizarse con el dispositivo. ${ }^{12}$ La simulación se desarrolló con una cámara endoscópica de $7 \mathrm{~mm}$ y seis diodos emisores de luz (LED) para Android ${ }^{\circledR}$ de dos metros de largo ensamblada a una rama recta $\mathrm{N} .^{\circ} 1$ de un laringoscopio Miller ${ }^{\circledast}$. Las imágenes de la intubación fueron recogidas en un teléfono celular con sistema operativo Android $10^{\circledR}$ con un programa específico para el endoscopio $\left(\right.$ AN98 $\left.{ }^{\circledR}\right)$ (Figura 1). Se utilizó un tubo endotraqueal N. ${ }^{\circ} 3,5$. La simulación se realizó con un cubo acrílico, que se utiliza como barrera de protección en la práctica asistencial durante la intubación de pacientes con enfermedad por el nuevo coronavirus (COVID-19).

Como medidas de resultado se utilizaron el tiempo de duración y el éxito del procedimiento. Se compararon las medias de los dos grupos mediante una prueba $t$, y se consideró un valor de $p<0,05$ con significación estadística. La medición del tiempo utilizado para el procedimiento se llevó a cabo con el cronómetro de un teléfono celular desde el momento en que el operador introdujo el videolaringoscopio en la boca del simulador hasta que se constató el ingreso del tubo endotraqueal en la laringe. Se definió como éxito del procedimiento a la confirmación de la expansión de los pulmones del simulador con el bolseo. Los operadores realizaron la práctica luego de firmar el consentimiento informado.

\section{RESULTADOS}

Realizaron el procedimiento de simulación 18 médicos. Se analizaron los datos de 15 de ellos, dado que en tres no se midieron los tiempos. Siete $(47 \%)$ eran del equipo de terapistas intensivos, seis $(40 \%)$ eran residentes de pediatría y dos $(13 \%)$ eran residentes de cuidados intensivos pediátricos. Se efectuaron, en total, 33 intentos, todos exitosos (Tabla 1).

Los operadores llevaron a cabo su primera intubación en un tiempo promedio de 116,4 segundos (IC95 \%: 84,8-148,0 [43-230]). 
El tiempo promedio del segundo intento fue 44,2 segundos (IC95\% 27,74-60,66 [16-111]). Al comparar el primero y el segundo intento de intubación, el tiempo disminuyó de forma significativa $(p=0,0001)$.

\section{DISCUSIÓN}

En el contexto de pandemia por COVID-19, los equipos de salud necesitan adquirir habilidades para llevar a cabo la labor cotidiana. Una habilidad técnica que requiere especial atención es la intubación de un paciente con sospecha o confirmación de COVID-19. Las guías elaboradas por diferentes sociedades de anestesia recomiendan la simulación para familiarizarse con los procesos planificados y reconocer dificultades no identificadas. Uno de los focos de aprendizaje es el de la protección del equipo de salud con dispositivos tecnológicos nuevos que requieren de cierta experticia como los videolaringoscopios. ${ }^{13}$

La ventaja de la videolaringoscopia radica en que aumenta la distancia entre la boca del paciente y la del operador. ${ }^{14}$
En un escenario de simulación, Begley y cols. midieron el tiempo utilizado por anestesistas para realizar una intubación endotraqueal con videolaringoscopia y cubo acrílico. Los operadores habían tenido un proceso de cinco minutos de instrucción previa y dos intentos de intubación antes de comenzar la simulación. La intubación con cubo duró 82,1 segundos (IC95 \%: 45,1-98,3 [30,8-180,0]) versus 42,9 segundos (IC95 \%: 32,9-46,9 [30,9.57,6]) sin cubo $(\mathrm{p}=0,002) .{ }^{15}$

En la simulación descrita en este trabajo, realizada con el videolaringoscopio de bajo costo, se midieron los tiempos de los dos primeros intentos. La media de intubación para el primer intento se alargó 34,3 segundos en comparación con el trabajo de Begley con videolaringoscopios comerciales; sin embargo, la duración del segundo intento, fue menos de la mitad del tiempo empleado en el primer intento.

El dispositivo permitió concretar la intubación de forma exitosa en todos los intentos, con acortamiento de la duración del procedimiento luego de una segunda práctica.

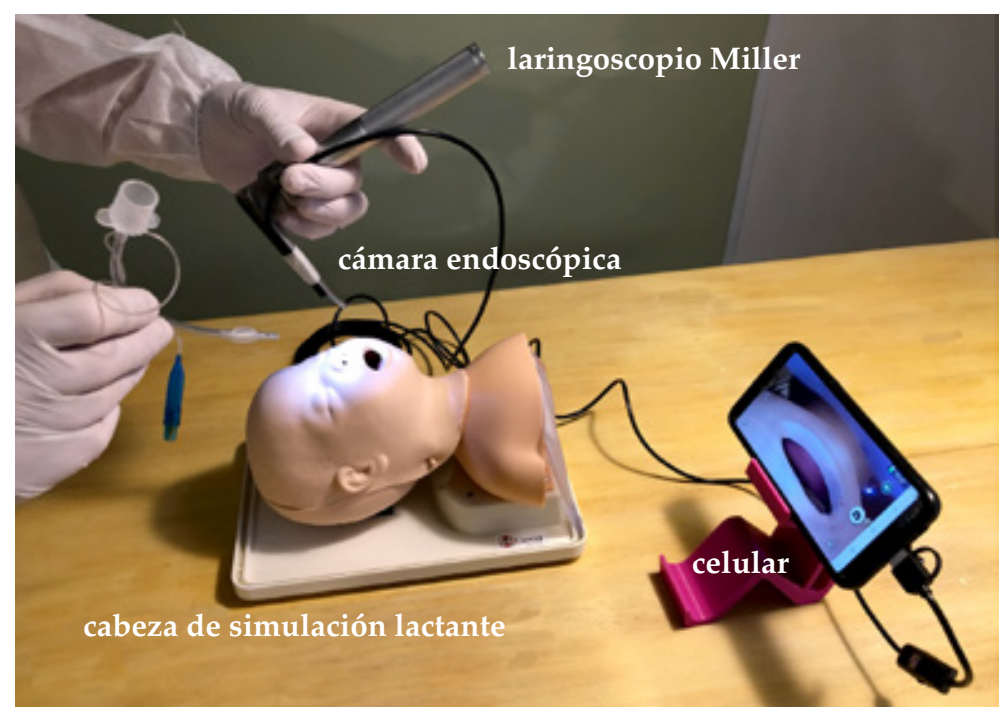

TABLA 1. Profesionales que participaron de la simulación

\begin{tabular}{llll}
\hline Profesionales & \multicolumn{2}{c}{ Intentos } \\
\hline 7 terapistas pediátricos & 7 terapistas: 2 intentos cada uno & 14 intentos \\
6 residentes de pediatría & 4 residentes: 2 intentos cada uno & 8 intentos \\
& & 2 residentes: 3 intentos cada uno & 2 intentos \\
& 2 residentes de terapia intensiva & 1 residente: 2 intentos & 3 intentos \\
Total & 15 profesionales & 1 residente: 3 intentos & 33 intentos \\
\hline
\end{tabular}


Esta experiencia pone la atención en una tecnología de alto costo de uso frecuente en países de ingresos altos y de escasa disponibilidad en economías como la de Argentina. El videolaringoscopio artesanal aparece como una alternativa a ese problema: su costo es más bajo (1900 pesos argentinos) y se puede reutilizar.

La cámara endoscópica se puede ensamblar a ramas de laringoscopios rectos o curvos. Su higiene es similar a la de cualquier laringoscopio convencional.

Se describe aquí una simulación que permitió a los pediatras familiarizarse con una tecnología nueva, que podría tener algún papel en la protección del equipo de salud y en la seguridad de los pacientes.

No se puede trasladar esta experiencia a una recomendación sobre el uso del dispositivo dado que aún no se ha probado su eficacia en el ámbito clínico pediátrico.

Se requiere investigación que aporte evidencia que responda a estos interrogantes.

\section{REFERENCIAS}

1. The Lancet. COVID-19: Protecting health-care workers. Lancet. 2020;395(10228):922.

2. Tran K, Cimon K, Severn M, Pessoa-Silva CL, et al. Aerosol generating procedures and risk of transmission of acute respiratory infections to healthcare workers: a systematic review. PLoS One. 2012;7(4):e35797.

3. Zuo MZ, Huang YG, Ma WH, Xue ZG, et al. Expert Recommendations for Tracheal Intubation in Critically ill Patients with Noval Coronavirus Disease 2019. Chin Med Sci J. 2020;35(2):105-9.
4. Orser BA. Recommendations for Endotracheal Intubation of COVID-19 Patients. Anesth Analg. 2020;130(5):1109-10.

5. Cook TM, El-Boghdadly K, McGuire B, McNarry AF, et al. Consensus guidelines for managing the airway in patients with COVID-19: Guidelines from the Difficult Airway Society, the Association of Anaesthetists the Intensive Care Society, the Faculty of Intensive Care Medicine and the Royal College of Anaesthetists. Anaesthesia. 2020;75(6):785-99.

6. Matava CT, Kovatsis PG, Lee JK, Castro P, et al. Pediatric Airway Management in COVID-19 Patients: Consensus Guidelines from the Society for Pediatric Anesthesia's Pediatric Difficult Intubation Collaborative and the Canadian Pediatric Anesthesia Society. Anesth Analg. 2020;131(1):61-73.

7. Balaban O, Tobias JD. Videolaryngoscopy in Neonates, Infants, and Children. Pediatr Crit Care Med. 2017;18(5): 477-85.

8. Grunwell JR, Kamat PP, Miksa M, Krishna A, et al. Trend and Outcomes of Video Laryngoscope Use Across PICUs. Pediatr Crit Care Med. 2017;18(8):741-9.

9. Grünberg G. Intubación nasotraqueal con "videolaringoscopio artesanal" en paciente con vía aérea dificultosa prevista. Anest Analg Reanim. 2012;25(2):55-60.

10. De Freitas J, Moreno J, Silva M. Prototipo de videolaringoscopio: Wi-Mac-Multivision. Rev Chil Anest 2020;49(2):262-70.

11. Karippacheril JG, Umesh G, Ramkumar V. Inexpensive video-laryngoscopy guided intubation using a personal computer: Initial experience of a novel technique. J Clin Monit Comput. 2014;28(3):261-4.

12. Simulación de Videolaringoscopía en lactante con COVID 19. [Consulta: 26 de mayo de 2020]. Disponible en: https: / / www.youtube.com/watch?v=7eAC6R17GuQ

13. Saito T, Taguchi A, Asai T. Videolaryngoscopy for tracheal intubation in patients with COVID-19. Br J Anaesth. 2020;125(3):e284-6.

14. Hall D, Steel A, Heij R, Eley A, et al. Videolaryngoscopy increases 'mouth-to-mouth' distance compared with direct laryngoscopy. Anaesthesia. 2020;75(6):822-3.

15. Begley JL, Lavery KE, Nickson CP, Brewster DJ. The aerosol box for intubation in coronavirus disease 2019 patients: an in-situ simulation crossover study. Anaesthesia. 2020;75(8):1014-21.

\title{
¿Es el electrocardiograma correctamente interpretado por médicos residentes de Pediatría? Experiencia previa y posterior a una intervención educacional en un hospital universitario
}

\author{
Are electrocardiograms correctly interpreted by pediatric residents? Experience \\ before and after an educational intervention in a teaching hospital
}

\author{
Dra. Georgina Bergero ${ }^{a}$ Dra. Melina J. Saavedra ${ }^{b}$ Dra. Marina Guglielmino ${ }^{a}$, Dra. Amanda R. Soto Pérez ${ }^{a}$, \\ Dr. Julián Llera ${ }^{a}$ y Dr. Julio Busaniche ${ }^{a}$
}

a. Servicio de Clínica Pediátrica, Departamento de Pediatría. b. ServiciodeCardiología Pediátrica, DepartamentodePediatría. Hospital Italiano de Buenos Aires, Ciudad Autónoma de Buenos Aires. Argentina.

Correspondencia:

Dra. Georgina Bergero: bergerogeorgina@gmail.com
Financiamiento: Ninguno.

Conflicto de intereses: Ninguno que declarar.

Recibido: 5-10-2020

Aceptado: 17-12-2020 